TRANSACTIONS OF THE

AMERICAN MATHEMATICAL SOCIETY

Volume 351, Number 7, Pages 2731-2743

S 0002-9947(99)02435-6

Article electronically published on February 23, 1999

\title{
A SPECIALITY THEOREM FOR COHEN-MACAULAY SPACE CURVES
}

\author{
ENRICO SCHLESINGER
}

\begin{abstract}
We prove a version of the Halphen Speciality Theorem for locally Cohen-Macaulay curves in $\mathbb{P}^{3}$. To prove the theorem, we strengthen some results of Okonek and Spindler on the spectrum of the ideal sheaf of a curve. As an application, we classify curves $C$ having index of speciality as large as possible once we fix the degree of $C$ and the minimum degree of a surface containing $C$.
\end{abstract}

\section{INTRODUCTION}

If $C$ is an algebraic curve in the projective space $\mathbb{P}^{3}$ over an algebraically closed field, the dimensions of the cohomology groups of the twisted ideal sheaf $\mathcal{I}_{C}(n)$ provide natural numerical invariants of $C$. The postulation of $C$ is defined as the sequence $\operatorname{dim} \mathrm{H}^{0}\left(\mathbb{P}^{3}, \mathcal{I}_{C}(n)\right)$, which counts the number of independent surfaces containing $C$ in each degree, while the speciality of $C$ is the sequence $\operatorname{dim} \mathrm{H}^{2}\left(\mathbb{P}^{3}, \mathcal{I}_{C}(n)\right)$ $=\operatorname{dim} \mathrm{H}^{1}\left(C, \mathcal{O}_{C}(n)\right)$, which measures how special the linear series $\left|\mathcal{O}_{C}(n)\right|$ are.

It is interesting in general to investigate what restrictions the postulation and the speciality impose on each other. The Halphen Speciality Theorem ([3], Theorem 1.2) is a result in this direction, as it gives a bound for $s(C)$, the smallest integer $n$ such that $\mathrm{H}^{0}\left(\mathbb{P}^{3}, \mathcal{I}_{C}(n)\right) \neq 0$, in terms of the degree of $C$ and of the index of speciality $e(C)$, which is the largest integer $n$ such that $\mathrm{H}^{1}\left(C, \mathcal{O}_{C}(n)\right) \neq 0$. In [3], the Halphen Speciality Theorem is proved for an integral curve in $\mathbb{P}^{3}$ (and more generally, for a codimension two integral subscheme of $\mathbb{P}^{N}$ ). However, it is often necessary to consider curves which may fail to be reduced or irreducible. We follow [8] in defining a curve to be a locally Cohen-Macaulay projective scheme of pure dimension one. In this paper, we prove a version of the Halphen Speciality Theorem for these more general curves in $\mathbb{P}^{3}$ :

Theorem 1.1 (cf. Theorem 6.5 below). Assume the ground field has characteristic zero. Let $C$ be a curve in $\mathbb{P}^{3}$ with index of speciality e. Suppose that no sub-curve $D$ of $C$ with $e(D)=e$ lies on a surface of degree $t-1$, and let $m$ be the minimum of $t$ and the integral part of $(e+4) / 2$. Then:

1. $\operatorname{deg} C \geq m(e+4-m)$ with equality holding if and only if $C$ is a specialization with constant cohomology of a complete intersection of two surfaces of degree $m$ and $e+4-m$;

2. $s(C) \leq \operatorname{deg} C-m(e+3-m)$.

Received by the editors April 20, 1997.

1991 Mathematics Subject Classification. Primary 14H50, 14F05, 14 F17.

(C)1999 American Mathematical Society 
If $C$ is integral, this implies that $s(C) \leq \max \{0, \operatorname{deg} C-n(e+4-n)\}+n$ for all positive integers $n$, which is the conclusion of the Halphen Speciality Theorem.

The theorem implies that for every curve $C$ we have $s(C) \leq \operatorname{deg}(C)-e(C)-$ 2. We give a partial classification of curves for which equality holds: if $s(C)=$ $\operatorname{deg}(C)-e(C)-2$ and $e(C) \geq 1$, we show that $C$ contains a plane curve $P$ of degree $\operatorname{deg}(C)-s(C)+1$; furthermore, if $H$ is the plane containing $P$ and $Y$ is the residual curve to $C$ with respect to $H$, then the support of $Y$ is either a line or two disjoint lines; on each line in its support, $Y$ is a quasiprimitive multiplicity structure (in the sense of [1]) which does not contain a planar double line.

The proof of the theorem is based on the study of the spectrum of the curve $C$, and in particular on Theorem 6.1 below which generalizes (in the case of curves) some results of Okonek and Spindler ([12], 2.4 and 4.1, [13], 2.3).

The paper is structured as follows: sections 2 and 3 contain the necessary background material and definitions. In section 4, building on [8], pp. 64-65, we prove a criterion, Proposition 4.2, for the existence of an elementary biliaison of negative height on a surface which is not necessarily integral. In section 5 , we prove a result, Proposition 5.3, on the cohomology of torsion free coherent sheaves on $\mathbb{P}^{3}$. It gives a bound for the number of generators of $\bigoplus_{n \in \mathbb{Z}} \mathrm{H}^{1}\left(\mathbb{P}^{3}, \mathcal{F}(n)\right)$ over the polynomial ring $k[x, y]$, where $x$ and $y$ are general linear forms, in terms of the spectrum of $\mathcal{F}$ as defined in [12]. In section 6 we prove our main results on the spectrum, and we deduce the speciality theorem.

Most of the results in this paper are taken from my Ph.D. thesis, and I wish to thank my adviser, Professor Robin Hartshorne, for his help and his kind support. I would also like to thank André Hirschowitz and the University of Nice for their kind hospitality during the period the first version of this paper was written.

\section{Preliminaries}

We work in the projective space $\mathbb{P}^{3}$ over an algebraically closed field $k$ of arbitrary characteristic. If $\mathcal{F}$ is a coherent sheaf on a closed subscheme $X \subseteq \mathbb{P}^{3}$, we let

$$
\mathrm{H}_{*}^{i}(X, \mathcal{F})=\bigoplus_{n \in \mathbb{Z}} \mathrm{H}^{i}(X, \mathcal{F}(n))
$$

and $h^{i}(X, \mathcal{F})=\operatorname{dim}_{k} \mathrm{H}^{i}(X, \mathcal{F})$.

We define a curve to be a locally Cohen-Macaulay projective scheme of pure dimension one. Given a curve $C \subset \mathbb{P}^{3}$, we fix homogeneous coordinates $x, y, z, w$ so that $x=y=0$ are the equations of a line $L$ disjoint from $C$, and we let $R=k[x, y, z, w]$ and $S=S_{L}=k[x, y]$. The ring $A_{C}=\mathrm{H}_{*}^{0}\left(C, \mathcal{O}_{C}\right)$ is CohenMacaulay of depth 2 over $R$ and $x, y$ form an $A_{C}$-regular sequence; hence $A_{C}$ is a free finitely generated graded $S$-module, and choosing a basis we obtain an isomorphism of $S$-modules:

$$
A_{C} \cong \bigoplus_{n \in \mathbb{Z}} S(-n)^{h_{C}(n)}
$$

where $h_{C}(n)$ is a sequence of nonnegative integers with finite support.

Given a numerical function $f$, we let $\partial f$ denote its first difference function $f(n)-$ $f(n-1)$. Then $h_{C}(n)=\partial^{2} h^{0} \mathcal{O}_{C}(n)$, so that the sequence $h_{C}(n)$ does not depend on the choice of $L$, and we call it the spectrum of $C$.

For convenience of notation, we also consider the spectrum of $C$ as the set $s p_{C}$ of integers with multiplicities in which the integer $n$ occurs $h_{C}(n)$ times, and we 
write $s p_{C}=\left\{n^{h_{C}(n)}\right\}$, or

$$
s p_{C}=\left\{n_{1}^{h_{C}\left(n_{1}\right)}, \ldots, n_{r}^{h_{C}\left(n_{r}\right)}\right\}
$$

if $n_{1}, \ldots, n_{r}$ are the integers for which $h_{C}(n)>0$. Finally we suppress the exponent $h_{C}(n)$ if it is equal to 1 .

Since the Hilbert polynomial of $C$ is linear, we have $h_{C}(n)=\partial^{2} h^{1} \mathcal{O}_{C}(n)$ as well, hence the information contained in the spectrum is precisely the speciality of the curve $C$. In particular, the degree of $C$ is equal to $\sum_{n \in \mathbb{Z}} h_{C}(n)$, while the arithmetic genus of $C$ is given by the formula:

$$
g(C)=\sum_{n \in \mathbb{Z}}(n-1) h_{C}(n)+1 .
$$

We denote by $\omega_{C}$ the Grothendieck dualizing sheaf of $C$, and we let $\Omega_{C}=$ $\mathrm{H}_{*}^{0}\left(C, \omega_{C}\right)$, the canonical module of $A_{C}$. If $L$ is a line disjoint from $C$ and $S=S_{L}$ is the polynomial subring of $R$ generated by the equations of $L$, then as $S$-modules

$$
\Omega_{C} \cong \operatorname{Hom}_{S}\left(A_{C}, S(-2)\right) \cong \bigoplus_{n \in \mathbb{Z}} S(-n)^{h_{C}(2-n)} .
$$

\section{The Principal Spectrum}

In this section we introduce the principal spectrum of $C$. It should be thought as a well behaved part of the spectrum: the spectrum is the set of integers which occur as degrees of the elements in a basis of $A_{C}$ over the ring $S$; as such it contains the set of integers which occur as degrees of the elements in a basis of the Rao module $M_{C}=\mathrm{H}_{*}^{1}\left(\mathbb{P}^{3}, \mathcal{I}_{C}\right)$. The principal spectrum is the difference of these two sets and behaves like the spectrum of an arithmetically Cohen-Macaulay curve.

Let $C \subset \mathbb{P}^{3}$ be a curve and let $L$ be a line which does not meet $C$. Tensoring the exact sequence $0 \rightarrow R_{C} \rightarrow A_{C} \rightarrow M_{C} \rightarrow 0$ over $S$ with $k \cong S /(x, y)$ we obtain a map $\phi: R_{C} \otimes k \rightarrow A_{C} \otimes k$. We define $P=P(C, L)$ to be the image of $\phi$.

Definition 3.1. The principal spectrum $s p_{C, L}$ of $C$ relative to $L$ is the set of integers with multiplicities $\left\{n^{h_{C, L}(n)}\right\}$ where

$$
h_{C, L}(n)=\operatorname{dim}_{k} P(C, L)_{n} .
$$

Hence $h_{C, L}(n)=h_{C}(n)-\mu_{C, L}(n)$ where $\mu_{C, L}=\operatorname{dim}_{k}\left(M_{C} \otimes k\right)_{n}$ is the number of generators in degree $n$ of $M_{C}$ over the ring $S$.

Note that the principal spectrum is never empty as the constant function one is a minimal generator of $A_{C}$ which maps to zero in the Rao module.

Remark 3.2. Let $T=R \otimes_{S} k \cong k[z, w]: P=P(C, L)$ is the quotient of $T$ by some proper homogeneous ideal $J$. Let $s=s(C, L)$ be the smallest integer $n$ such that $J_{n} \neq 0$. Then $h_{C, L}(n)=n+1$ for $0 \leq n \leq s-1, h_{C, L}(n) \geq h_{C, L}(n+1)$ for $n \geq s-1$, and $h_{C, L}(n)=0$ for $n>>0$ and $n<0$. Since the ground field $k$ is infinite, multiplication by a general linear form $z \in T_{1}$ has maximal rank on $P$, that is to say, $z: P_{n} \rightarrow P_{n+1}$ is injective for $n \leq s-2$ and surjective for $n \geq s-1$.

Consider now an inclusion of curves $C \subseteq Y \subset \mathbb{P}^{3}$. In general, it is not true that the spectrum of $C$ is a subset of the spectrum of $Y$. However, it is true that the principal spectrum of $C$ is contained in that of $Y$ : 
Proposition 3.3. Let $C$ and $Y$ be curves in $\mathbb{P}^{3}$, and suppose that $C \subseteq Y$. Let $L$ be a line which doesn't meet $Y$. Then $h_{C, L}(n) \leq h_{Y, L}(n)$ for all integers $n$.

Proof. The inclusion $C \subseteq Y$ induces a surjective map $P(Y, L) \rightarrow P(C, L)$.

There is a dense open subset $U$ of the Grassmannian of lines in $\mathbb{P}^{3}$ such that the principal spectrum of $C$ relative to any $L \in U$ is as large as possible and constant on $U$. Thus we can define the principal spectrum of $C$ as the principal spectrum of $C$ relative to a line $L \in U$.

\section{Variation of the Spectrum under Biliaison}

In this section we study the variation of the spectrum under an elementary biliaison. We borrow the following definition of an elementary biliaison from [6], page 318 ; an equivalent definition is given in [8].

Let $C$ and $D$ be curves in $\mathbb{P}^{3}$. We say that $D$ is obtained from $C$ by an elementary biliaison (or double linkage) of height $l$, for some $l \in \mathbb{Z}$, if there is a surface $F$ containing $C$ and $D$, such that $D$ is linearly equivalent to $C+l H$ as generalized divisors on $F$, where $H$ is a plane section of $F$. Furthermore, if $l$ is positive (resp. negative), we say that $C$ bilinks up (resp. down) on $F$. Note that saying that $D$ is linearly equivalent to $C+l H$ on $F$ means precisely that there is an isomorphism $\mathcal{I}_{D, F} \cong \mathcal{I}_{C, F}(-l)$.

Proposition 4.1. Let $C$ and $D$ be curves in $\mathbb{P}^{3}$, and suppose that $D$ is obtained from $C$ by an elementary biliaison of height $l \geq 1$ on the surface $F$. Then

$$
h_{D}(n)=h_{C}(n-l)+h_{F_{l}}(n)
$$

where $F_{l}$ denotes the complete intersection of $F$ with a surface of degree $l$ which meets $F$ properly.

Furthermore, if $L$ is a line which meets neither $C$ nor $D$, the formula holds with $h_{D, L}$ and $h_{C, L}$ in place of $h_{D}$ and $h_{C}$.

Proof. Since $\mathrm{H}_{*}^{1}\left(\mathbb{P}^{3}, \mathcal{I}_{F}\right)=\mathrm{H}_{*}^{2}\left(\mathbb{P}^{3}, \mathcal{I}_{F}\right)=0$ and $\mathcal{I}_{D, F} \cong \mathcal{I}_{C, F}(-l)$, we have an isomorphism

$$
M_{D} \cong M_{C}(-l)
$$

between the Rao modules of $C$ and $D$. Therefore it is enough to prove our statement for the spectrum, and for this it suffices to note the equality:

$$
h^{0} \mathcal{O}_{D}(n)=h^{0} \mathcal{O}_{C}(n-l)+h^{0} \mathcal{O}_{F_{l}}(n) .
$$

The following proposition gives a criterion for bilinking down a curve $C$ on a surface $Q$ in $\mathbb{P}^{3}$; we argue as in [8], pp. 64-65, but we go a little further.

Proposition 4.2 (Biliaison Trick). Let $C$ be a curve in $\mathbb{P}^{3}$. Let $Q$ be a surface of degree s containing $C$. Suppose there exists a nonzero element $\xi \in \mathrm{H}^{0} \omega_{C}(-m)$ such that $f \cdot \xi \neq 0$ for every form $f$ of degree $l<s$ which divides the equation of $Q$. Then either there is a curve $C_{0} \subset Q$ such that $C$ is obtained from $C_{0}$ by an elementary biliaison on $Q$ of height $m+4-s$, or $m+4-s \geq 1$ and $C$ is the complete intersection of $Q$ with a surface of degree $m+4-s$. 
Proof. Let $h=m+4-s$. As in [8], Lemma III.2.4 p.63, we look at the exact sequence:

$$
0 \rightarrow \mathrm{H}^{0} \mathcal{O}_{Q}(-h) \rightarrow \operatorname{Hom}\left(\mathcal{I}_{C, Q}(h), \mathcal{O}_{Q}\right) \rightarrow \mathrm{H}^{0} \omega_{C}(-m) \rightarrow 0
$$

which shows that $\xi$ lifts to a nonzero morphism $u: \mathcal{I}_{C, Q}(h) \rightarrow \mathcal{O}_{Q}$.

If $u$ is injective, we define $C_{0}$ by $\operatorname{Im} u=\mathcal{I}_{C_{0}, Q}$ and conclude as in [8], Proposition III.2.3, that $C_{0}$ is either empty, in which case $C$ is the complete intersection of $Q$ with a surface of degree $h \geq 1$, or a curve, in which case $C$ is obtained from $C_{0}$ by an elementary biliaison of height $h$.

Suppose $u$ is not injective. The kernel of $u$ has the form $\mathcal{I}_{D, Q}(h)$ for some subscheme $D$ of $Q$. Note that $D$ can't be all of $Q$ because $u$ is not injective. On the other hand, $D$ must contain some component of $Q$ : otherwise, the image of $u$, whose associated points are among those of $\mathcal{O}_{Q}$, would be zero. Let $F$ be the two-dimensional component of $D$. Since $\operatorname{Im} u$ is annihilated by $\mathcal{I}_{D, Q}$, we have $\mathcal{I}_{F, Q} \operatorname{Im} u=0$ at the generic points of $Q$ and hence everywhere. Thus, if $f$ is the equation of $F$, we have $f \cdot \xi=0$, which contradicts our hypothesis since $F$ is a proper subscheme of $Q$. Hence $u$ must be injective, and we are done.

We now give an example of how one can use Proposition 4.2 even when $Q$ is not integral; for an application see [11].

Proposition 4.3. Let $Q$ be a surface of degree 2 in $\mathbb{P}^{3}$, and let $C \subset Q$ be a curve which is not a complete intersection and has spectrum $\left\{n^{h_{C}(n)}\right\}$. Then $C$ bilinks down on $Q$ if and only if $h_{C}(1) \geq 2$.

Proof. One direction is easy: if $C$ bilinks down on $Q$, then by Proposition 4.1 the spectrum of $C$ contains $\left\{0,1^{2}\right\}$ hence $h_{C}(1) \geq 2$.

Conversely, suppose that $h_{C}(1) \geq 2$. Then

$$
h^{0} \omega_{C}(1)=\sum_{k \geq 1} k \cdot h_{C}(k) \geq 2 .
$$

If there is a linear form $h$ that kills $\mathrm{H}^{0} \omega_{C}(1)$, then $h$ kills $\mathrm{H}^{0} \omega_{C}(l)$ for all $l \leq 1$.

We let $H$ be the plane defined by $h$.

From the exact sequence:

$$
0 \rightarrow \omega_{C \cap H} \rightarrow \omega_{C} \stackrel{h}{\rightarrow} \omega_{C}(1)
$$

we see that $\mathrm{H}^{0}\left(\omega_{C \cap H}(n)\right) \cong \mathrm{H}^{0}\left(\omega_{C}(n)\right)$ for $n \leq 1$.

If we let $P$ denote the largest Cohen-Macaulay curve contained in $C \cap H$, then $\omega_{P}=\omega_{C \cap H}$ because $P$ and $C \cap H$ coincide in dimension one (note that $C \cap H$ has dimension one since $h$ kills a nonzero submodule of $\left.\Omega_{C}\right)$. Thus we have $\mathrm{H}^{0}\left(\omega_{P}(n)\right) \cong$ $\mathrm{H}^{0}\left(\omega_{C}(n)\right)$ for $n \leq 1$ and this implies $h_{C}(n)=h_{P}(n)$ for $n \geq 1$.

Now $P$ is a plane curve, so its spectrum is $\{0,1, \ldots, \operatorname{deg} P-1\}$, and we conclude that $h_{C}(l)=1$ for all $1 \leq l \leq e(C)+2$. In particular, $h_{C}(1)=1$ which is a contradiction. Hence $h$ does not kill all of $\mathrm{H}^{0} \omega_{C}(1)$, and therefore we can choose a nonzero element $\xi \in \mathrm{H}^{0} \omega_{C}(1)$ such that $h \cdot \xi \neq 0$ for all linear forms $h$ dividing the equation of $Q$. Since $C$ is not a complete intersection, Proposition 4.2 implies that $C$ is obtained from some curve $C_{0}$ by an elementary biliaison on $Q$ of height $h=-1+4-2=1$, hence $C$ bilinks down on $Q$.

Corollary 4.4. Let $Q$ be a surface of degree 2 in $\mathbb{P}^{3}$, and let $C \subset Q$ be a curve which is not ACM. Then $C \subset Q$ is minimal in its biliaison class (cf. [8], p. 71) 
if and only if its spectrum satisfies $h_{C}(1) \leq 1$; and if $C$ is not minimal, it bilinks down on $Q$.

Proof. Quite generally, if $C$ is not ACM and is not minimal, then according to [8] $C$ can be deformed with constant spectrum and Rao module to a curve $D$ which bilinks down on some surface of degree at least two. By Proposition 4.1 we have $s p_{C}=s p_{D} \supseteq\left\{0,1^{2}\right\}$ hence $h_{C}(1) \geq 2$. Thus any non-ACM curve with $h_{C}(1) \leq 1$ is minimal. The rest of the statement follows from Proposition 4.3.

Remark 4.5. In general, it is not known if a non-minimal curve $C$ bilinks down on some surface $Q \supset C$ : see [8], 5.4, p.93.

\section{A Remark on the Cohomology of Torsion Free Sheaves}

Let $\mathcal{F}$ be a torsion free coherent sheaf on $\mathbb{P}^{3}$. One knows that $\mathcal{F}$ is (locally) a first syzygy, hence the singularity locus

$$
S_{1}(\mathcal{F})=\left\{P \in \mathbb{P}^{3}: \operatorname{projdim}_{P} \mathcal{F}_{P} \geq 1\right\}
$$

is a closed subscheme of $\mathbb{P}^{3}$ of dimension at most one; similarly,

$$
S_{2}(\mathcal{F})=\left\{P \in \mathbb{P}^{3}: \operatorname{projdim}_{P} \mathcal{F}_{P} \geq 2\right\}
$$

is closed of dimension at most zero.

If $H$ is a plane missing $S_{2}(\mathcal{F})$ and meeting $S_{1}(\mathcal{F})$ only at finitely many points, the restriction $\mathcal{F}_{H}$ of $\mathcal{F}$ to $H$ is a torsion free $\mathcal{O}_{H}$ module. If $L$ is a line skew to $S_{1}(\mathcal{F})$, the restriction $\mathcal{F}_{L}$ of $\mathcal{F}$ to $L$ is a locally free $\mathcal{O}_{L}$ module. Thus, if $L$ is a general line and $H$ is a general plane through $L, \mathcal{F}_{L}$ is locally free and $\mathcal{F}_{H}$ is torsion free.

Now suppose $\mathcal{F}$ has rank $r$ and $L$ is a line not meeting $S_{1}(\mathcal{F})$; the restriction $\mathcal{F}_{L}$ splits in the form $\mathcal{F}_{L} \cong \bigoplus_{i=1}^{r} \mathcal{O}_{L}\left(a_{i}\right)$ for some integers $a_{i}$,

$$
a_{\mathcal{F}}(L)=\left(a_{1}, \ldots, a_{r}\right) .
$$

We define $b=b(\mathcal{F}, L)$ to be the largest of the integers $a_{i}$, and $a=a(\mathcal{F}, L)$ to be the smallest.

Before we proceed, we need to introduce some more notation. As above, we denote by $R$ the homogeneous coordinate ring of $\mathbb{P}^{3}$. Given a graded $R$-module $M$, the vector space $M^{*}=\bigoplus_{n \in \mathbb{Z}} \operatorname{Hom}_{k}\left(M_{-n}, k\right)$ has a structure of graded $R$-module, with grading given by $M_{n}^{*}=\operatorname{Hom}_{k}\left(M_{-n}, k\right)$ and multiplication defined by the formula $(f \phi)(m)=\phi(f m)$ for homogeneous elements $f \in R, \phi \in M^{*}$ and $m \in M$.

Definition 5.1 (cf. [5], page 170). Let $T=k\left[z_{1}, \ldots, z_{N}\right]$ be a polynomial ring over the field $k$, and let $M$ be a graded $T$-module. If $f_{1}, \ldots, f_{r}$ are forms in $T$, we write $M /\left(f_{1}, \ldots, f_{r}\right)$ for $M \otimes_{T} T /\left(f_{1}, \ldots, f_{r}\right)$. A sequence of linear forms $x_{1}, \ldots, x_{r}$ is called $M$-regular up to degree $a$ if the multiplication map

$$
x_{i}:\left(M /\left(x_{1}, \ldots, x_{i-1}\right)\right)_{n-1} \rightarrow\left(M /\left(x_{1}, \ldots, x_{i-1}\right)\right)_{n}
$$

is injective for all $n \leq a$ and all $i=1, \ldots, r$.

We define $M_{1}(\mathcal{F})=\mathrm{H}_{*}^{1}(X, \mathcal{F})$ and $M_{2}(\mathcal{F})=\left(\mathrm{H}_{*}^{2}(X, \mathcal{F})\right)^{*}$.

Lemma 5.2. Let $\mathcal{F}$ be a torsion free coherent sheaf on $\mathbb{P}^{3}$. Let $L$ be a line not meeting $S_{1}(\mathcal{F})$, and let $H$ be a plane through $L$ which does not meet $S_{2}(\mathcal{F})$. Fix equations $x=0$ for $H$ and $x=y=0$ for $L$, and let $a=a(\mathcal{F}, L)$ and $b=b(\mathcal{F}, L)$. Then 
1. $x, y$ is $M_{1}(\mathcal{F})$-regular up to degree $-b-1$.

2. $x, y$ is $M_{2}(\mathcal{F})$-regular up to degree $a+3$.

3. Multiplication by $y: \mathrm{H}^{1} \mathcal{F}_{H}(l-1) \rightarrow \mathrm{H}^{1} \mathcal{F}_{H}(l)$ is injective for $l \leq-b-1$ and surjective for $l \geq-a-1$.

4. $\mathrm{H}^{0} \mathcal{F}_{H}(l)=\mathrm{H}^{0} \mathcal{F}(l)=0$ for $l \leq-b-1$.

5. $\mathrm{H}^{2} \mathcal{F}_{H}(l)=\mathrm{H}^{3} \mathcal{F}(l-1)=0$ for $l \geq-a-2$.

Proof. We first show that $h^{0} \mathcal{F}_{H}(l)=0$ if $l \leq-b-1$. It is clear that $h^{0} \mathcal{F}_{L}(l)=0$ for $l \leq-b-1$ as $\mathcal{F}_{L} \cong \bigoplus_{i=1}^{r} \mathcal{O}_{L}\left(a_{i}\right)$; our hypotheses imply that $\mathcal{F}_{H}$ is torsion free, hence we have an exact sequence $0 \rightarrow \mathcal{F}_{H}(-1) \rightarrow \mathcal{F}_{H} \rightarrow \mathcal{F}_{L} \rightarrow 0$ which tells us that the function $l \mapsto h^{0} \mathcal{F}_{H}(l)$ is constant for $l \leq-b-1$. But for $l \ll 0, h^{0} \mathcal{F}_{H}(l)=0$ as $\mathcal{F}_{H}$ is torsion free. Hence $h^{0} \mathcal{F}_{H}(l)=0$ if $l \leq-b-1$. A similar argument shows that $h^{0} \mathcal{F}(l)=0$ for $l \leq-b-1$.

Next we prove that $x, y$ form an $M_{1}(\mathcal{F})$-regular sequence up to degree $-b-1$. Observe that the kernel of multiplication by $x: \mathrm{H}^{1} \mathcal{F}(l-1) \rightarrow \mathrm{H}^{1} \mathcal{F}(l)$ is a quotient of $\mathrm{H}^{0} \mathcal{F}_{H}(l)$; since the latter vanishes for $l \leq-b-1$, multiplication by $x$ on $M_{1}$ is injective in degrees $\leq-b-1$. Now consider the exact sequence

$$
0 \longrightarrow \mathcal{F}_{H}(-1) \stackrel{y}{\longrightarrow} \mathcal{F}_{H} \longrightarrow \mathcal{F}_{L} \longrightarrow 0 .
$$

Because of the vanishing of $\mathrm{H}^{0} \mathcal{F}_{L}(l)$, we see that multiplication by

$$
y: \mathrm{H}^{1} \mathcal{F}_{H}(l-1) \rightarrow \mathrm{H}^{1} \mathcal{F}_{H}(l)
$$

is injective for $l \leq-b-1$; as $M_{1}(\mathcal{F}) / x \subseteq \mathrm{H}_{*}^{1} \mathcal{F}_{H}$ we conclude that $x, y$ form an $M_{1}(\mathcal{F})$-regular sequence up to degree $-b-1$. The proof of the other statement is analogous.

If $L$ is the line $x=y=0$, we let $\mu_{1}(\mathcal{F}, L)(n)=\operatorname{dim}_{k}\left(M_{1}(\mathcal{F}) /(x, y)\right)_{n}$ and $\mu_{2}(\mathcal{F}, L)(n)=\operatorname{dim}_{k}\left(M_{2}(\mathcal{F}) /(x, y)\right)_{n}$ for all integers $n$. Note that, if $M_{1}(\mathcal{F})_{n}=0$ for $n \ll 0$, that is, if projdim $\mathcal{F} \leq 1$, then $\mu_{1}(\mathcal{F}, L)(n)$ is the number of minimal generators of $M_{1}(\mathcal{F})$ over $S=k[x, y]$ in degree $n$.

Proposition 5.3. Let $\mathcal{F}$ be a coherent torsion free sheaf on $\mathbb{P}^{3}$, and let $L$ be a line on which $\mathcal{F}$ is locally free. Then

$$
\begin{array}{ll}
\partial^{2} h^{1} \mathcal{F}(n)=\mu_{1}(n) \geq \mu_{2}(2-n) & \text { if } \quad n \leq-b(\mathcal{F}, L)-1 \\
\partial^{2} h^{2} \mathcal{F}(n)=\mu_{2}(2-n) \geq \mu_{1}(n) & \text { if } \quad n \geq-a(\mathcal{F}, L)-1 .
\end{array}
$$

Proof. We can choose equations $x=y=0$ for $L$ such that the plane $H$ of equation $x$ does not meet $S_{1}(\mathcal{F})$.

Looking at $0 \rightarrow \mathcal{F}(-1) \rightarrow \mathcal{F} \rightarrow \mathcal{F}_{H} \rightarrow 0$ and taking cohomology we find an exact sequence

$$
0 \rightarrow M_{1} / x \rightarrow M_{H} \rightarrow\left(M_{2} / x\right)^{*}(-1) \rightarrow 0 .
$$

Tensoring with $R / y$ we obtain a six term exact sequence

$$
\begin{aligned}
0 & \rightarrow \operatorname{Tor}_{1}^{R}\left(M_{1} / x, R / y\right) \rightarrow \operatorname{Tor}_{1}^{R}\left(M_{H}, R / y\right) \rightarrow \operatorname{Tor}_{1}^{R}\left(\left(M_{2} / x\right)^{*}(-1), R / y\right) \\
& \rightarrow M_{1} /(x, y) \rightarrow M_{H} / y \rightarrow\left(M_{2} / x\right)^{*} \otimes R / y(-1) \rightarrow 0 .
\end{aligned}
$$

Since for any graded $R$-module $N$ we have $\operatorname{Tor}_{1}^{R}\left(N^{*}, R / y\right) \cong(N / y)^{*}(-1)$, the dimensions of the $n^{\text {th }}$ graded components of the third and fourth terms in the above sequence are $\mu_{2}(2-n)$ and $\mu_{1}(n)$ respectively.

By Lemma 5.2, $\operatorname{Tor}_{1}^{R}\left(M_{H}, R / y\right)$ vanishes for $n \leq-b(\mathcal{F}, L)-1$, hence in this range $\mu_{1}(n) \geq \mu_{2}(2-n)$. On the other hand, by Lemma 5.2 again, $(x, y)$ form 
an $M_{1}$-regular sequence up to degree $-b(\mathcal{F}, L)-1$, hence in this range $\mu_{1}(n)=$ $\partial^{2} h^{1} \mathcal{F}(n)$. For $n \geq-a(\mathcal{F}, L)-1$ the proof is similar.

\section{The Speciality Theorem}

In this section we use the machinery developed so far to prove Theorem 6.3 below. As a corollary, we obtain a version for Cohen-Macaulay curves of the Halphen Speciality Theorem ([3], Theorem 1.2).

Let $C$ be a curve in $\mathbb{P}^{3}$. We denote by $s(C)$ the smallest integer $n$ such that there is a surface of degree $n$ containing $C$.

To each nonzero homogeneous element $\xi$ of the canonical module $\Omega_{C}=\mathrm{H}_{*}^{0}\left(C, \omega_{C}\right)$ we associate the subcurve $C_{\xi}$ of $C$ defined by the ideal

$$
I_{\xi}=\{f \in R: f \xi=0\} .
$$

Since $R / I_{\xi}$ is a submodule of $\Omega_{C}$, whose associated points correspond to the irreducible components of $C$, the ideal $I_{\xi}$ is saturated, and $C_{\xi}$ is locally CohenMacaulay of pure dimension one. If we define $\tau(\xi)$ to be the smallest degree of a nonzero form which annihilates $\xi$, then $\tau(\xi)=s\left(C_{\xi}\right)$ because $I_{\xi}$ is saturated.

Note that the canonical module $\Omega_{C_{\xi}}$ can be identified with the submodule of $\Omega_{C}$ annihilated by $I_{\xi}$. To see this, let $L$ be a line skew to $C$, and apply the functor $\operatorname{Hom}_{S_{L}}\left(-, \Omega_{S_{L}}\right)$ to the exact sequence

$$
0 \rightarrow I_{\xi} / I_{C} \rightarrow R_{C} \rightarrow R_{C_{\xi}} \rightarrow 0
$$

In particular, $\xi$ is an element of $\Omega_{C_{\xi}}$, and therefore $e\left(C_{\xi}\right) \geq-\operatorname{deg}(\xi)$.

Theorem 6.1. Assume the ground field has characteristic zero. Let $C$ be a curve in $\mathbb{P}^{3}$ and let $\xi \in \Omega_{C}$ be homogeneous of degree $-p$ with $\tau(\xi)=\tau$. If $L$ is a general line and $1 \leq t \leq \tau$, then

$$
h_{C}(n) \geq t+\mu_{C, L}(n) \quad \text { for } t-1 \leq n \leq p+3-t
$$

where $\mu_{C, L}(n)=\operatorname{dim}_{k}\left(M_{C} \otimes_{S} S / m_{S}\right)_{n}$ is the number of minimal generators in degree $n$ of the Rao module $M_{C}$ over the polynomial ring $S=S_{L}$.

Proof. Let $m$ be the minimum of $\tau$ and the integral part of $(p+4) / 2$. Let $X$ be the complete intersection of two surfaces of degree $m$ and $p+4-m$; $X$ has the largest spectrum among complete intersections $Y$ satisfying $s(Y) \leq \tau$ and $e(Y) \leq p$, namely,

$$
h_{X}(n)= \begin{cases}n+1 & \text { if } 0 \leq n \leq m-1, \\ m & \text { if } m-1 \leq n \leq p+3-m, \\ p+3-m & \text { if } p+3-m \leq n \leq p+2 .\end{cases}
$$

We have to prove that the principal spectrum of $C$ with respect to a general line $L$ contains the (principal) spectrum of $X$.

Since $C_{\xi} \subseteq C$, it is enough to prove the lemma for $C_{\xi}$, that is, we may assume that the annihilator of $\xi$ is the homogeneous ideal $I_{C}$, and in particular that $s(C)=\tau$.

We have two cases to consider. First suppose that $\tau \leq p+4-\tau$ so that $m=\tau$. Pick a surface $Q$ of degree $\tau$ containing $C$. Since $\xi$ is not killed by any form of degree smaller than $\tau$, we can use Proposition 4.2 to conclude that either $C$ is obtained by an elementary biliaison of height $h=p+4-\tau$ on $Q$ from some curve $C_{0}$, or $C$ is a complete intersection of two surfaces of degrees $\tau$ and $p+4-\tau$. Now we conclude using Proposition 4.1. Note that so far we have not used the characteristic zero hypothesis. 
The second case we have to consider is $\tau>p+4-\tau$. In this case, $m$ is equal to the integral part of $(p+4) / 2$. We write $2 m=p+4+c_{1}$ with $c_{1}=0$ or -1 .

Now $\mathrm{H}^{0} \omega_{C}(-p) \cong \operatorname{Ext}_{\mathcal{O}_{\mathbb{P} 3}}^{1}\left(\mathcal{I}_{C}(m), \mathcal{O}_{\mathbb{P}^{3}}\left(c_{1}-m\right)\right)$ and $\xi$ corresponds under this isomorphism to an extension $0 \rightarrow \mathcal{O}_{\mathbb{P}^{3}}\left(c_{1}-m\right) \rightarrow \mathcal{F} \rightarrow \mathcal{I}_{C}(m) \rightarrow 0$ where $\mathcal{F}$ is a rank two torsion free sheaf with first Chern class $c_{1}$ (in fact, $\mathcal{F}$ is even reflexive). We wish to show that $\mathcal{F}$ is stable. Applying the functor $\operatorname{Hom}\left(-, \mathcal{O}_{\mathbb{P}^{3}}\left(c_{1}\right)\right)$ to the exact sequence above, we obtain

$$
0 \rightarrow \mathrm{H}^{0} \mathcal{O}\left(c_{1}-m\right) \rightarrow \mathrm{H}^{0} \mathcal{F}^{\vee}\left(c_{1}\right) \rightarrow \mathrm{H}^{0} \mathcal{O}(m) \stackrel{\xi}{\rightarrow} \mathrm{H}^{0} \omega_{C}(m-p) .
$$

We have $m-c_{1} \geq m \geq 1$ because $p \geq-2$; on the other hand, by construction, $m \leq \tau-1=\tau(\xi)-1$, hence $\mathrm{H}^{0} \mathcal{O}(m) \stackrel{\xi}{\rightarrow} \mathrm{H}^{0} \omega_{C}(m-p)$ is injective. Therefore $\mathrm{H}^{0} \mathcal{F}^{\vee}\left(c_{1}\right)=0$.

Now observe that $\mathcal{F}^{\vee \vee} \cong \mathcal{F}^{\vee}\left(c_{1}\right)$ by [4], Proposition 1.10, because $\mathcal{F}^{\vee}$ is a rank two reflexive sheaf with first Chern class $-c_{1}$. It follows from [4], Lemma 3.1, that $\mathcal{F}^{\vee \vee}$ is stable in the sense of Mumford-Takemoto.

Now we use the characteristic zero hypothesis: by the Theorem of GrauertMülich ([2], Theorem 12 and its Corollary), the restriction of $\mathcal{F}^{\vee \vee}$, and hence of $\mathcal{F}$, to a general line $L$ is isomorphic to $\mathcal{O}_{L} \bigoplus \mathcal{O}_{L}\left(c_{1}\right)$. We fix such a general line $L$.

We define the spectrum of $\mathcal{F}$ (cf. [12]) by the formula

$$
h_{\mathcal{F}}(n)= \begin{cases}\partial^{2} h^{1} \mathcal{F}(n) & \text { if } n \leq-1 \\ \partial^{2} h^{2} \mathcal{F}(n) & \text { if } n \geq 0\end{cases}
$$

Note that by Proposition 5.3 we have $h_{\mathcal{F}}(n) \geq \mu_{1}(\mathcal{F}, L)(n)$ for all integers $n$, and from the isomorphism $\mathrm{H}_{*}^{1}\left(\mathbb{P}^{3}, \mathcal{F}\right) \cong \mathrm{H}_{*}^{1}\left(\mathbb{P}^{3}, \mathcal{I}_{C}(m)\right)$ it follows that $\mu_{1}(\mathcal{F}, L)(n)=$ $\mu_{C, L}(n+m)$. Hence to finish the proof of the theorem, it is enough to show that for all integers $n$ we have $h_{C}(n+m)=h_{\mathcal{F}}(n)+h_{X}(n+m)$ where $X$ is the complete intersection of two surfaces of degrees $m$ and $m-c_{1}=p+4-m$.

For the complete intersection $X$ we have

$$
h_{X}(n+m)= \begin{cases}\max \left(0, m-c_{1}-n-1\right) & \text { if } n \geq 0 \\ \max (0, n+m+1) & \text { if } n \leq-1 .\end{cases}
$$

By Lemma $5.2 \mathrm{H}^{3}\left(\mathbb{P}^{3}, \mathcal{F}(n)\right)=0$ if $n \geq-2$. Thus for $n \geq-2$ we have an exact sequence

$$
0 \rightarrow \mathrm{H}^{2} \mathcal{F}(n) \rightarrow \mathrm{H}^{2} \mathcal{I}_{C}(n+m) \rightarrow \mathrm{H}^{3} \mathcal{O}\left(n-m-c_{1}\right) \rightarrow 0
$$

from which we deduce that for $n \geq 0$

$$
\begin{aligned}
h_{C}(n+m) & =\partial^{2} h^{2} \mathcal{I}_{C}(n+m) \\
& =\partial^{2} h^{2} \mathcal{F}(n)+\max \left(0, m-c_{1}-n-1\right) \\
& =h_{\mathcal{F}}(n)+h_{X}(n+m) .
\end{aligned}
$$

On the other hand, we have $h^{0} \mathcal{I}_{C}(n+m)=0$ for $n \leq-1$ because $h^{0} \mathcal{F}(n)=$ $h^{0} \mathcal{F}^{\vee \vee}(n)=0$ for $n \leq-1$. Hence for $n \leq-1$ we have

$$
\begin{aligned}
h_{\mathcal{F}}(n) & =\partial^{2} h^{1} \mathcal{F}(n)=\partial^{2} h^{1} \mathcal{I}_{C}(n+m) \\
& =\partial^{2} h^{0} \mathcal{O}_{C}(n+m)-\partial^{2} h^{0} \mathcal{O}_{\mathbb{P}^{3}}(n+m) \\
& =h_{C}(n+m)-\max (0, n+m+1) \\
& =h_{C}(n+m)-h_{X}(n+m)
\end{aligned}
$$

and this concludes the proof. 
Definition 6.2. Let $C$ be a curve in $\mathbb{P}^{3}$ with index of speciality $e$. We define $\tau(C)$ to be the largest of the integers $\tau(\xi)$ as $\xi$ varies in $\mathrm{H}^{0} \omega_{C}(-e)$. We always have $\tau(C) \leq s(C)$, with equality holding if $C$ is an integral curve.

An immediate consequence of Theorem 6.1 is the following:

Theorem 6.3. Assume the ground field has characteristic zero. Let $C$ be a curve in $\mathbb{P}^{3}$ with invariants $e=e(C)$ and $\tau=\tau(C)$. If $L$ is a general line and $1 \leq t \leq \tau$, then

$$
h_{C}(n) \geq t+\mu_{C, L}(n) \quad \text { for } t-1 \leq n \leq e+3-t .
$$

Remark 6.4. For an integral curve $C$, the statement of Theorem 6.3 with the term $\mu_{C, L}(n)$ deleted remains true in any characteristic as in the proof we can use Hartshorne's characteristic free theory of the spectrum for rank 2 reflexive sheaves [4].

As a consequence of Theorem 6.3, we obtain a version of the Halphen Speciality Theorem for Cohen-Macaulay curves:

Theorem 6.5. Assume the ground field has characteristic zero. Let $C$ be a curve in $\mathbb{P}^{3}$ with invariants $e=e(C)$ and $\tau=\tau(C)$. Then for all positive integers $m$ satisfying $m \leq \min \{\tau,(e+4) / 2\}$ we have:

1. $\operatorname{deg} C \geq m(e+4-m)$ with equality holding if and only if $C$ is a specialization with constant cohomology of a complete intersection of two surfaces of degree $m$ and $e+4-m$;

2. $s(C) \leq \operatorname{deg} C-m(e+3-m)$.

Proof. Theorem 6.3 tells us that the principal spectrum of $C$ contains the spectrum of the complete intersection $X$ of two surfaces of degrees $m$ and $e+4-m$; in particular, $\operatorname{deg} C \geq \operatorname{deg} X=m(e+4-m)$.

Suppose equality holds: then in the chain of inclusions $s p_{X} \subseteq s p_{C, L} \subseteq s p_{C}$ the two finite sets on the left and on the right have the same number of elements, hence we have equality everywhere. Equality on the right tells us that $C$ is ACM. For an ACM curve, the spectrum is equivalent to the postulation, and the set of ACM curves with given postulation is irreducible. This proves the first statement.

To prove 2, we fix a general line $L$ of equations $x=y=0$ and a linear form $z$ mapping to a general linear form of $R /(x, y)$. The determinant trick (cf. [14]) shows that $s(C)$ is less than or equal to the number of generators of $A_{C}$ over $k[x, y, z]$. Thus it is enough to show that the number of generators of $A_{C}$ over $k[x, y, z]$ is at most $\operatorname{deg} C-m(e+3-m)$. To this end, we look at the exact sequence defining the principal spectrum:

$$
0 \rightarrow P(C, L) \rightarrow A_{C} \otimes_{S} k \rightarrow M_{C} \otimes_{S} k \rightarrow 0
$$

where $S=k[x, y]$, and we let $\phi: P(C, L) \rightarrow P(C, L)(1)$ denote multiplication by $z$. The number of generators of $A_{C}$ over $S$ is equal to $\operatorname{dim} A_{C} \otimes_{S} k=\operatorname{deg} C$, hence the number of generators of $A_{C}$ over $k[x, y, z]$ is at most $\operatorname{deg} C$ minus the dimension of the image of $\phi$. Now by Remark $3.2 \phi_{n}: P(C, L)_{n} \rightarrow P(C, L)_{n+1}$ has maximal rank for all $n$, so that $\operatorname{dim} \operatorname{Im}\left(\phi_{n}\right)=\min \left\{\operatorname{dim} P_{n}, \operatorname{dim} P_{n+1}\right\}$.

By Theorem 6.3 we have $\operatorname{dim} P_{n} \geq h_{X}(n)$, where $X$ is the complete intersection of two surfaces of degree $m$ and $e+4-m$. Thus

$$
\operatorname{dim} \operatorname{Im}\left(\phi_{n}\right) \geq \min \left\{h_{X}(n), h_{X}(n+1)\right\}=h_{Y}(n)
$$


where $Y$ is the complete intersection of two surfaces of degrees $m$ and $e+3-m$. Summing over $n$ we find $\operatorname{dim} \operatorname{Im}(\phi) \geq \operatorname{deg} Y=m(e+3-m)$, hence the number of generators of $A_{C}$ over $k[x, y, z]$ is at most $\operatorname{deg} C-m(e+3-m)$, and we are done.

The following corollary implies the Halphen Speciality Theorem for integral curves in $\mathbb{P}^{3}$. Gruson and Peskine proved the speciality theorem for codimension two integral subschemes of $\mathbb{P}^{N}$ for any $N \geq 3$ : see [3], Theorem 1.2.

Corollary 6.6 (Halphen-Gruson-Peskine). Assume the ground field has characteristic zero. Let $C$ be a curve in $\mathbb{P}^{3}$ with index of speciality e. Suppose that $s(C)=\tau(C)$ (this is the case for $C$ integral). Then

$$
s(C) \leq \max \{0, \operatorname{deg} C-n(e+4-n)\}+n
$$

for all positive integers $n$.

Proof. We have several possibilities to consider: if $n \leq \min \{s(C),(e+4) / 2\}$, by Theorem 6.5 we have $\operatorname{deg} C \geq n(e+4-n)$ and

$$
s(C) \leq \operatorname{deg}(C)-n(e+3-n)=\max \{0, \operatorname{deg} C-n(e+4-n)\}+n .
$$

If $n \geq s(C)$, the statement is obvious. If $(e+4) / 2<n<s(C)$, we have two cases: in the first case we have $n \leq e+4$ and we can set $m=e+4-n$ and use Theorem 6.5 as above, keeping in mind that $m<n$ by construction. In the second case we have $n \geq e+4$, so that the right hand side $\max \{0, \operatorname{deg} C-n(e+4-n)\}+n$ is at least $\operatorname{deg} C$ which is always an upper bound for $s(C)$.

Theorem 6.5 shows that for a curve $C \subset \mathbb{P}^{3}$ we always have $s(C) \leq \operatorname{deg}(C)-$ $e(C)-2$. This is true in any characteristic, and for all positive integers $s$ and $d$ satisfying $s \leq d$ there are curves $C$ satisfying $\operatorname{deg}(C)=d, s(C)=s, e(C)=d-s-2$ and containing a plane curve of degree $d-s+1$ [14]. These curves are interesting because of the maximum genus problem [14], and among them (when $s=2$ ) are the extremal curves of $[10,9]$ which have the largest Rao function among curves with fixed degree and genus.

We can now show that all curves $C$ satisfying $s(C)=\operatorname{deg}(C)-e(C)-2$ and $e(C) \geq 1$ contain a plane curve of degree $d-s+1$. Recall (see [7]) that, if $C$ is a curve with a component contained in the plane $H$, the residual scheme $Y=\operatorname{Res}_{H}(C)$ to $C$ with respect to $H$ is defined by the exact sequence

$$
0 \rightarrow \mathcal{I}_{Y, \mathbb{P}^{3}}(-1) \stackrel{h}{\rightarrow} \mathcal{I}_{C, \mathbb{P}^{3}} \rightarrow \mathcal{I}_{C \cap H, H} \rightarrow 0
$$

where $h$ is the equation of $H$. Note that $Y$ is a subscheme of $C$, and that the associated points of $Y$ are among the associated points of $C$, as one sees from the injective map $\mathcal{O}_{Y}(-1) \stackrel{h}{\rightarrow} \mathcal{O}_{C}$; hence $Y$ doesn't have zero dimensional associated points, and is a curve in our sense.

Proposition 6.7. Assume the ground field has characteristic zero. Let $C$ be a curve in $\mathbb{P}^{3}$ with invariants $d=\operatorname{deg}(C), s=s(C)$ and $e=e(C)$. Suppose $s=$ $d-e-2$ and $e \geq 1$. Then

1. $C$ contains a plane curve $P$ of degree $d-s+1($ so that $e(P)=e(C))$;

2. if $H$ is the plane containing $P$ and $Y=\operatorname{Res}_{H}(C)$ is the residual curve,

$$
s(Y)=\operatorname{deg}(Y)=s-1 ;
$$

3. we have $h_{C}(n)=1$ for $2 \leq n \leq e+2$. 
Proof. We first show that $\tau(C)$ must be one: otherwise, in Theorem 6.5 we could take $m=2$ and conclude $s \leq d-2 e-2<d-e-2$, a contradiction. Hence $\tau(C)=1$ so that $C$ contains a plane curve $P$ with $e(P)=e$. We have $\operatorname{deg}(P)=e(P)+3=$ $d-s+1$.

Since $\operatorname{deg}(P) \geq 4$, there is a unique plane $H$ containing $P$. Furthermore, since $e(P)=e(C), P$ is the largest curve contained in $C \cap H$.

Let $Y=\operatorname{Res}_{H}(C)$.

From the exact sequence

$$
0 \rightarrow \mathcal{I}_{Y}(-1) \rightarrow \mathcal{I}_{C} \rightarrow \mathcal{I}_{C \cap H, H} \rightarrow 0,
$$

we see that $\operatorname{deg}(Y)=\operatorname{deg}(C)-\operatorname{deg}(P)=s-1$ and that $s(Y) \geq s-1$. Since for all curves $D$ we have $s(D) \leq \operatorname{deg}(D)$, we conclude that $s(Y)=\operatorname{deg}(Y)=s-1$.

Next we look at the exact sequence $0 \rightarrow \mathcal{O}_{Y}(-1) \rightarrow \mathcal{O}_{C} \rightarrow \mathcal{O}_{C \cap H} \rightarrow 0$ from which we obtain $0 \rightarrow \Omega_{P} \rightarrow \Omega_{C} \rightarrow \Omega_{Y}(1)$ since $P$ is equal to $C \cap H$ in dimension one. As $Y$ satisfies $s(Y)=\operatorname{deg}(Y)$, we have $e(Y)=-2$, therefore $\Omega_{P}$ and $\Omega_{C}$ are equal in degrees $\leq 0$. This implies $h_{C}(n)=h_{P}(n)=1$ for $2 \leq n \leq e+2$, and we are done.

Remark 6.8. One can prove that, if $Y$ is a curve satisfying $s(Y)=\operatorname{deg}(Y)$, the support of $Y$ is either a line or two disjoint lines. If $X$ is a connected component of $Y$, then $s(X)=\operatorname{deg}(X)$ and $X$ is a quasiprimitive multiplicity structure on a line (in the sense of [1]) which does not contain a planar double structure.

\section{REFERENCES}

1. C. Banica and O. Forster. Multiplicity structures on space curves. In Lefschetz Centennial Conference, Contemporary Mathematics 58, pages 47-64. AMS, 1986. MR 88c:32018

2. O. Forster, A. Hirschowitz, and M. Schneider. Type de scindage généralisé pour les fibrés stables. In A. Hirschowitz, editor, Vector bundles and differential equations, Proceedings, Nice, 1979, Progress in Mathematics 7. Birkhäuser, Boston-Basel-Stuttgart, 1980. MR 81k:14016

3. L. Gruson and C. Peskine. Genre des courbes de l'espace projectif. In Algebraic Geometry, Tromsø 1977, Lecture Notes in Math 687, pages 31-59. Springer-Verlag, 1978. MR 81e:14019

4. R. Hartshorne. Stable reflexive sheaves. Math. Ann., 254:121 -176, 1980. MR 82b:14011

5. R. Hartshorne. Stable reflexive sheaves II. Invent. Math., 66:165-190, 1982. MR 83e:14012

6. R. Hartshorne. Generalized divisors on Gorenstein schemes. K-Theory, 8:287-339, 1994. MR 95k:14008

7. R. Hartshorne and A. Hirschowitz. Droites en position générale dans l'espace projectif. In Algebraic Geometry, Proc. La Rabida 1981, Lecture Notes in Math 961, pages 169-189. Springer-Verlag, 1982. MR 85e:14043

8. M. Martin-Deschamps and D. Perrin. Sur la classification des courbes gauches. Astérisque 184-185. Société Mathématique de France, 1990. MR 91h:14039

9. M. Martin-Deschamps and D. Perrin. Sur les bornes du module de Rao. C.R. Acad. Sci. Paris, Série I, 137:1159-1162, 1993. MR 95e:14023

10. M. Martin-Deschamps and D. Perrin. Le schéma de Hilbert des courbes gauches localement Cohen-Macaulay n'est (presque) jamais réduit. Ann. Sc. E.N.S., $4^{e}$ Série, 29:757-785, 1996. MR 98a:14003

11. S. Nollet. Subextremal curves. Manuscripta Math., 94:303-317, 1997. CMP 98:05

12. C. Okonek and H. Spindler. Das Spektrum torsionsfreier Garben I. Manuscripta Math., 47:187-228, 1984. MR 85h:14008 
13. C. Okonek and H. Spindler. Das Spektrum torsionsfreier Garben II. In Seminar on Deformations, Lecture Notes in Math 1165, pages 211-234. Springer-Verlag, 1985. MR 87d:14012

14. E. Schlesinger. A New Proof of a Theorem of Beorchia on the Genus of Space Curves . Mat. Nach. 196 (1988), 197-203.

Dipartimento di Matematica, Università di Trento, 38050 Povo (Trento), Italy

E-mail address: schlesin@science.unitn.it 